Institute of Chemistry \& Chemical Technology

\title{
Two new alkaloids from the aerial parts of Caryopteris mongolica Bunge.
}

\author{
M.Dumaa $^{1}$, Ya.Gerelt-Od ${ }^{1}$, Zh.Puzhao ${ }^{2}$, L.Yinggang ${ }^{2}$, S.Javzan ${ }^{1}$, \\ D.Selenge ${ }^{1}$, G.Zhang ${ }^{2}$
}

${ }^{1}$ Institute of Chemistry and Chemical Technology, MAS, Ulaanbaatar, Mongolia

${ }^{2}$ Chengdu Institute of Biology, CHAS, Chengdu, China

\begin{abstract}
Two new alkaloids moncaryopterine A and moncaryopterine B were isolated from the aerial parts of Caryopteris mongolica Bunge. by the column chromatography and HPLC methods. Molecular structures of them were elucidated by MS, ${ }^{1} \mathrm{H},{ }^{13} \mathrm{C}$, HSQC, HMBC, ${ }^{1} \mathrm{H} /{ }^{1} \mathrm{H}$ COSY, and ${ }^{1} \mathrm{H} /{ }^{1} \mathrm{H}$ NOESY NMR methods.
\end{abstract}

\section{Keywords: Caryopteris mongolica Bunge. Verbenaceae, alkaloids,} moncaryopterine A, moncaryopterine B

\section{INTRODUCTION}

C aryopteris mongolica Bunge. is a deciduous shrub and belongs to the Verbenaceae family. It is widely distributed throughout the Mongolian territory [1]. In addition, this plant species grows in some provinces of Hebei, Shanxi of Inner Mongolia and Gansu, China [2]. In fact, Caryopteris mongolica is only species grown in Mongolia, whereas about 16 other species are discovered in different places of the world.

In Mongolian traditional medicine aerial parts of $C$. mongolica have been used for the treatment of haemorrhage, chronic bronchitis and for an increasing of the muscle strength and urinary excretion [3]. In Chinese folk medicine Caryopteris terniflora has been used as antipyretic, expectorant and for the treatment of tuberculosis, rheumatism and cold [4]. Some species of Caryopteris are cultivated for a decoration arrangement and ornamental purposes.

Previous chemical investigations of Caryopteris mongolica showed the presence of essential oils, mono and sesquiterpenoids [5], hypolaetin7-glucoside [6], iridiod glucosides and steriods [7, 8, 9]. Moreover, from other species of
Caryopteris iridoids, steroid glucosides, phenylethanoids, diterpenoids, phenolic acids, $\alpha$-caryopterone, a new pyranojuglone, clandonoside and its acetylated derivatives have been isolated, respectively $[10,11,12,13,14]$. To the best of our knowledge there are no data on alkaloids in all species of Caryopteris. However, we are reporting here of the molecular structure elucidation of two alkaloids isolated from the aerial parts of $C$. mongolica.

\section{EXPERIMENTAL}

Plant material. The aerial parts of Caryopteris mongolica Bunge. were collected from Terelj Mountain chains, vicinity of Ulaanbaatar, during the flowering period in August 2010. Dr. B. Mandakh, Institute of Botany, MAS has identified the plant species and voucher specimen was deposited at the Herbariums of the Natural Products Chemistry Laboratory, Institute of Chemistry and Chemical Technology of the Mongolian Academy of Sciences.

Extraction and isolation of alkaloids. The air dried and powdered aerial parts $(3.3 \mathrm{~kg})$ of Caryopteris mongolica were extracted with 94\% ethanol at room temperature for 3 times. 
The ethanol extract was evaporated to dryness and treated with $2.5 \% \mathrm{HCl},(\mathrm{pH}=1-2)$. The aqueous acidic solution was extracted successively with petroleum ether and chloroform, respectively. Then the aqueous residue solution was adjusted to $\mathrm{pH}=9-10$ by aq. $\mathrm{NH}_{4} \mathrm{OH} \quad(25 \%)$ and extracted with dichloromethane, which was evaporated under reduced pressure to give $1.5 \mathrm{~g}$ of crude total alkaloids, $(0.048 \%)$. The crude total alkaloids isolated from $C$. mongolica were then carried out through sephadex LH-20. The column (150 $\mathrm{cm} \times 4 \mathrm{~cm})$ was eluted with chloroformmethanol (1:1) and the elutes were controlled by TLC, detected by the Dragendorff reagent and Iodine. Fractions with similar quality were combined and totally collected 6 subfractions from A to F- A-0.149 g, B-0.128 g, C-0.153 g, D-0.246 g, E-0.443 $\mathrm{g}$ and F-0.043 g, respectively.

The fraction D $(0.246 \mathrm{~g})$ was subjected to Silica gel (10 g), $\mathrm{GF}_{254}, 200-300$ mesh (Qingdao Haiyang Chemical Corporation, China), the column $(18 \mathrm{~cm} \times 2 \mathrm{~cm})$ was eluted with chloroform-methanol mixtures $50: 1$ to $1: 1$ to give 7 subfractions $D_{1}-D_{7}$. The fraction $D_{1}(77$ $\mathrm{mg}$ ) was separated on a Semipreparative HPLC using an $\mathrm{MeOH}-\mathrm{H}_{2} \mathrm{O}(10: 1$, v/v) solvent system at a flow rate of $3 \mathrm{~mL} / \mathrm{min}$ and the peaks were detected at $208 \mathrm{~nm}$. The compounds at retention times 29 and $33 \mathrm{~min}$. were isolated and marked as $\mathbf{1},(5 \mathrm{mg})$ and $\mathbf{2 ,}(8 \mathrm{mg})$, respectively.

Structure elucidation of alkaloids. Mass spectra (ESIMS) was obtained on Finnigan$\mathrm{LCQ}^{\mathrm{DECA}}$ mass spectrometer. 1D NMR: ${ }^{1} \mathrm{H}$, $(600 \mathrm{MHz}) ;{ }^{13} \mathrm{C}(150 \mathrm{MHz})$ and 2D NMR: HMQC, HMBC, ${ }^{1} \mathrm{H} /{ }^{1} \mathrm{H}$ COSY, and NOESY spectra were recorded on an Avance Bruker 600 spectrometer with TMS as an internal standard. The chemical shifts $(\delta)$ are reported in ppm and the coupling constants (J) are given in hertz, (Hz). Column chromatography (CC) was performed on self-packed open column with Sephadex LH-20 from Sweden and Silica gel $\mathrm{GF}_{254}$, 200-300 mesh from Qingdao Haiyang Chemical Co., Ltd. (QHCC, PR China) and Silica gel $\mathrm{GF}_{254}$ 0-40 mesh (QHCC) for thin layer chromatography (TLC) analyses were used, respectively. Spots on TLC plates detected under UV lamp at 254 or $365 \mathrm{~nm}$ and visualized by spraying with the Dragendorff reagent, pure iodine $\left(\mathrm{J}_{2}\right)$ and $8 \%$ solution of phosphomolybdic acid in ethanol. Semipreparative HPLC was equipped with a Perkin-Elmer Series 200 pump, a Perkin-Elmer Series UV/vis detector. For HPLC analysis $20 \mu \mathrm{L}$ samples were injected.

\section{RESULTS AND DISCUSSION}

The compound $\mathbf{1}$ is colorless needles, with the molecular formula $\mathrm{C}_{18} \mathrm{H}_{16} \mathrm{~N}_{2} \mathrm{O}_{2}$ from ESIMS $\left(\mathrm{m} / \mathrm{z} 315.1104[\mathrm{M}+\mathrm{Na}]^{+}\right)$. The ${ }^{1} \mathrm{H}$ NMR data of the compound 1 in $\mathrm{CDCl}_{3}$ (Table 1) showed that 6 aromatic protons, 4 aliphatic protons and methyl resonances at $\delta 1.52$ and at $\delta 1.67 \mathrm{ppm}$, respectively.

Table $1 .{ }^{1} \mathrm{H}$ and ${ }^{13} \mathrm{C}$ NMR data for compounds $\mathbf{1}$ and $\mathbf{2}$ in $\mathrm{CDCl}_{3},(\delta$ in ppm, $\mathrm{J}$ in $\mathrm{Hz})$

\begin{tabular}{|c|c|c|c|c|}
\hline$: \stackrel{0}{0}$ & $\begin{array}{c}\delta \mathrm{H}, \mathrm{m}, \mathrm{J} \\
(\mathrm{Hz})\end{array}$ & $\begin{array}{c}\delta \mathrm{H}, \mathrm{m}, \mathrm{J} \\
(\mathrm{Hz})\end{array}$ & $\delta \mathrm{C}$ & $\delta \mathrm{C}$ \\
\hline & 1 & 2 & 1 & 2 \\
\hline 1 & - & - & $\begin{array}{c}204.91 \\
\text { (CO) }\end{array}$ & $\begin{array}{c}205.32 \\
\text { (CO) }\end{array}$ \\
\hline 2 & - & - & $141.91,(\mathrm{C})$ & $141.85,(\mathrm{C})$ \\
\hline 4 & $\begin{array}{c}8.75, \mathrm{~d}, \\
(1 \mathrm{H}), \\
\mathrm{J}=4.8\end{array}$ & $\begin{array}{c}8.76, \mathrm{~d}, \\
(1 \mathrm{H}), \\
\mathrm{J}=4.8\end{array}$ & $\begin{array}{c}149.03 \\
(\mathrm{CH})\end{array}$ & $\begin{array}{c}149.00 \\
(\mathrm{CH})\end{array}$ \\
\hline 5 & $\begin{array}{c}7.56, \mathrm{~d}, \\
(1 \mathrm{H}), \\
\mathrm{J}=4.8\end{array}$ & $\begin{array}{c}7.58, \mathrm{~d}, \\
(1 \mathrm{H}), \\
\mathrm{J}=4.8\end{array}$ & $\begin{array}{c}116.18 \\
(\mathrm{CH})\end{array}$ & $\begin{array}{c}116.38 \\
(\mathrm{CH})\end{array}$ \\
\hline 6 & - & - & $152.80,(\mathrm{C})$ & $152.33,(\mathrm{C})$ \\
\hline $6 a$ & - & - & $44.21,(\mathrm{C})$ & $43.96,(\mathrm{C})$ \\
\hline 7 & $\begin{array}{c}2.54, \mathrm{~d}, \\
(1 \mathrm{H}), \\
\mathrm{J}=18 \\
2.81, \mathrm{~d}, \\
(1 \mathrm{H}), \\
\mathrm{J}=18\end{array}$ & $\begin{array}{c}2.49, \mathrm{~d}, \\
(1 \mathrm{H}), \mathrm{J}=18 \\
2.60, \mathrm{~d}, \\
(1 \mathrm{H}), \mathrm{J}=18\end{array}$ & $\begin{array}{l}48.97, \\
\left(\mathrm{CH}_{2}\right)\end{array}$ & $\begin{array}{l}48.24, \\
\left(\mathrm{CH}_{2}\right)\end{array}$ \\
\hline 8 & - & - & $\begin{array}{c}203.70 \\
(\mathrm{CO})\end{array}$ & $\begin{array}{c}203.68 \\
\text { (CO) }\end{array}$ \\
\hline 9 & - & - & $142.20,(\mathrm{C})$ & $142.11,(\mathrm{C})$ \\
\hline 11 & $\begin{array}{c}8.70, \mathrm{~d}, \\
(1 \mathrm{H}), \\
\mathrm{J}=4.8\end{array}$ & $\begin{array}{c}8.70, \mathrm{~d}, \\
(1 \mathrm{H}), \\
\mathrm{J}=4.8\end{array}$ & $\begin{array}{c}148.97 \\
(\mathrm{CH})\end{array}$ & $\begin{array}{c}148.99 \\
(\mathrm{CH})\end{array}$ \\
\hline 12 & $\begin{array}{c}\text { 7.46, d, } \\
(1 \mathrm{H}), \\
\mathrm{J}=4.8\end{array}$ & $\begin{array}{c}\text { 7.47, d, } \\
(1 \mathrm{H}), \\
\mathrm{J}=4.8\end{array}$ & $\begin{array}{c}116.10 \\
(\mathrm{CH})\end{array}$ & $\begin{array}{c}116.19 \\
(\mathrm{CH})\end{array}$ \\
\hline 13 & - & - & $150.24,(\mathrm{C})$ & $150.23,(\mathrm{C})$ \\
\hline 14 & $\begin{array}{c}3.41, \mathrm{~m}, \\
(1 \mathrm{H})\end{array}$ & $\begin{array}{c}2.99, \mathrm{~m}, \\
(1 \mathrm{H})\end{array}$ & $36.19,(\mathrm{CH})$ & $\begin{array}{c}36.16 \\
(\mathrm{CH})\end{array}$ \\
\hline $\begin{array}{c}14 \\
\mathrm{a}\end{array}$ & $\begin{array}{l}2.66, d, \\
(1 \mathrm{H}), \\
\mathrm{J}=3.6\end{array}$ & $\begin{array}{l}2.77, \mathrm{~d} \\
(1 \mathrm{H}), \\
\mathrm{J}=3.6\end{array}$ & $63.25,(\mathrm{CH})$ & $\begin{array}{l}62.19 \\
(\mathrm{CH})\end{array}$ \\
\hline 15 & $\begin{array}{c}8.94, \mathrm{~s} \\
(1 \mathrm{H})\end{array}$ & $\begin{array}{c}8.87, \mathrm{~s}, \\
(1 \mathrm{H})\end{array}$ & $\begin{array}{c}149.12 \\
(\mathrm{CH})\end{array}$ & $\begin{array}{c}149.43 \\
(\mathrm{CH})\end{array}$ \\
\hline 16 & $\begin{array}{c}8.97, \mathrm{~s}, \\
(1 \mathrm{H})\end{array}$ & $\begin{array}{c}9.02, \mathrm{~s}, \\
(1 \mathrm{H})\end{array}$ & $\begin{array}{c}148.05 \\
(\mathrm{CH})\end{array}$ & $\begin{array}{c}148.08, \\
(\mathrm{CH})\end{array}$ \\
\hline 17 & $\begin{array}{c}1.67, \mathrm{~s}, \\
(3 \mathrm{H}),(- \\
\left.\mathrm{CH}_{3}\right)\end{array}$ & $\begin{array}{c}1.85, \mathrm{~s}, \\
(3 \mathrm{H}),(- \\
\left.\mathrm{CH}_{3}\right)\end{array}$ & $\begin{array}{c}26.97,(- \\
\left.\mathrm{CH}_{3}\right)\end{array}$ & $\begin{array}{c}27.58,(- \\
\left.\mathrm{CH}_{3}\right)\end{array}$ \\
\hline 18 & $\begin{array}{l}1.52, \mathrm{~d}, \\
(3 \mathrm{H}), \\
\mathrm{J}=7.2\end{array}$ & $\begin{array}{l}1.30, \mathrm{~d}, \\
(3 \mathrm{H}), \\
\mathrm{J}=7.2\end{array}$ & $\begin{array}{c}22.16,(- \\
\left.\mathrm{CH}_{3}\right)\end{array}$ & $\begin{array}{c}21.87,(- \\
\left.\mathrm{CH}_{3}\right)\end{array}$ \\
\hline
\end{tabular}


In addition ${ }^{13} \mathrm{C}$ NMR data revealed the presence of 18 carbons including two methyl groups and two keto groups. An analysis of the ${ }^{1} \mathrm{H}$ NMR spectrum (Table 1) in conjunction with the HSQC data confirmed that $\delta$ : two methyls at 22.16 and $26.97 \mathrm{ppm}$, a methylene protons at $48.97 \mathrm{ppm}$, eight methines at $36.19,63.25$, $116.10,116.18,148.05,149.03,149.12$, and $148.97 \mathrm{ppm}$, respectively. Two keto groups $\delta$ : at 203.70, 204.91 and five quaternary carbons at $44.21,141.91,142.20,150.24,152.80 \mathrm{ppm}$, respectively, were found. HMBC cross-peaks from 2 methyl groups $\delta$ : at $1.52 \mathrm{ppm}, \mathrm{d}, \mathrm{J}=7.2$ $\mathrm{Hz}, 3 \mathrm{H}$ to $\mathrm{C}-36.19, \mathrm{C}-63.25$ and $\mathrm{C}-150.24$ and 1.67 ppm, s, $3 \mathrm{H}$ to $\mathrm{C}-44.21, \mathrm{C}-48.97, \mathrm{C}-63.25$ and $\mathrm{C}-152.80 \mathrm{ppm}$, respectively. Two protons of methylene $\delta$ : at $2.54, \mathrm{~d}, \mathrm{~J}=18,1 \mathrm{H}$, and 2.81 , $\mathrm{d}, \mathrm{J}=18,1 \mathrm{H},\left(\mathrm{CH}_{2}\right)$ to $\mathrm{C}-26.97, \mathrm{C}-63.25, \mathrm{C}-$ 152.80 and C-203.70 ppm, respectively. All interpretations of the HMBC spectrum for compound 1 showed in Table 2.
${ }^{1} \mathrm{H}$ COSY correlations of the $\mathrm{H}-4 / \mathrm{H}-5$ and $\mathrm{H}-$ $11 / \mathrm{H}-12$ spin systems in the aromatic region and $\mathrm{H}-18 / \mathrm{H}-14 \mathrm{a} / \mathrm{H}-14$ coupling systems. The following HMBC correlations showed: H-18/C14, C-13 and C-14a; H-14a/C-6a, C-7, C-14a respectively. Furthermore, the configuration of compound 1 was established by the NOESY correlations were of $\mathrm{H}-14 \mathrm{a} / \mathrm{H}-17, \mathrm{H}-14 ; \mathrm{H}-$ $14 / \mathrm{H}-17$, H-7. Thus, the structure of the compound 1 was determined as (6aR,14R,14aS)-6a,14-dimethyl-6a, 7,14,14atetrahydro-2,6:9,13-di(metheno)azocino[5,4e]azecine-1,8-dione. This is naturally a new alkaloid isolated and identified for the first time from $C$. mongolica grown in the Mongolian flora, thus given a trivial name "moncaryopterine A", (1). The compound $\mathbf{2}$ is colorless needles, with the molecular formula $\mathrm{C}_{18} \mathrm{H}_{16} \mathrm{~N}_{2} \mathrm{O}_{2}$ from ESIMS $(\mathrm{m} / \mathrm{z}, 315.1111[\mathrm{M}+$ $\mathrm{Na}]^{+}$). The molecular formula and molecular weight, ${ }^{1} \mathrm{H},{ }^{13} \mathrm{C}$ NMR data of compound 2 (Table 1) were identical with the compound $\mathbf{1}$.

Table 2. Interpretation of the HMBC spectrum for compound $\mathbf{1}$, $(\delta$ in ppm, $\mathrm{J}$ in $\mathrm{Hz}$

\begin{tabular}{|c|c|c|c|c|}
\hline $\begin{array}{c}1.52, \mathrm{~d}, \mathrm{~J}=7.2,3 \mathrm{H},\left(\mathrm{CH}_{3}\right) \\
\mathrm{C}-22.16,\end{array}$ & $\mathrm{C}-36.19,(\mathrm{CH})$ & $\mathrm{C}-63.25,(\mathrm{CH})$ & $\mathrm{C}-150.24,(\mathrm{C})$ & \\
\hline $\begin{array}{c}1.67, \mathrm{~S}, 3 \mathrm{H},\left(\mathrm{CH}_{3}\right) \\
\mathrm{C}-26.97\end{array}$ & $\mathrm{C}-44.21,(\mathrm{C})$ & $\mathrm{C}-48.97,\left(\mathrm{CH}_{2}\right)$ & $\mathrm{C}-63.25,(\mathrm{CH})$ & $\mathrm{C}-152.80,(\mathrm{C})$ \\
\hline $\begin{array}{c}2.54, \mathrm{~d}, \mathrm{~J}=18,1 \mathrm{H},\left(\mathrm{CH}_{2}\right) \\
2.81, \mathrm{~d}, \mathrm{~J}=18,1 \mathrm{H} \\
\mathrm{C}-48.97\end{array}$ & $\begin{array}{c}\mathrm{C}-26.97,\left(\mathrm{CH}_{3}\right) \\
\text { and C-44.21(C) }\end{array}$ & $\mathrm{C}-63.25,(\mathrm{CH})$ & $\mathrm{C}-152.80,(\mathrm{C})$ & $\mathrm{C}-203.70,(\mathrm{CO})$ \\
\hline $\begin{array}{c}2.66, \mathrm{~d}, \mathrm{~J}=3.6,1 \mathrm{H},(\mathrm{CH}) \\
\mathrm{C}-63.25\end{array}$ & $\begin{array}{c}\mathrm{C}-44.21,(\mathrm{C}) \text { and } \\
\text { C-26.97, }\left(\mathrm{CH}_{3}\right)\end{array}$ & $\mathrm{C}-36.19,(\mathrm{CH})$ & $\mathrm{C}-48.97,\left(\mathrm{CH}_{2}\right)$ & $\mathrm{C}-204.91,(\mathrm{CO})$ \\
\hline $\begin{array}{c}7.46, \mathrm{~d}, \mathrm{~J}=4.8,1 \mathrm{H},(\mathrm{CH}) \\
\mathrm{C}-116.10\end{array}$ & $\mathrm{C}-148.97,(\mathrm{C})$ & $150.24,(\mathrm{C})$ & & \\
\hline $\begin{array}{c}7.56, \mathrm{~d}, \mathrm{~J}=4.8,1 \mathrm{H},(\mathrm{CH}) \\
\mathrm{C}-116.18\end{array}$ & $\mathrm{C}-152.80,(\mathrm{C})$ & $149.03,(\mathrm{CH})$ & & \\
\hline $\begin{array}{c}8.70, \mathrm{~d}, \mathrm{~J}=4.8,1 \mathrm{H},(\mathrm{CH}) \\
\mathrm{C}-148.97\end{array}$ & $\mathrm{C}-116.10$ & $\mathrm{C}-142.20,(\mathrm{C})$ & & \\
\hline $\begin{array}{c}8.75, \mathrm{~d}, \mathrm{~J}=4.8,1 \mathrm{H},(\mathrm{CH}) \\
\mathrm{C}-149.03\end{array}$ & $\mathrm{C}-116.18$ & $\mathrm{C}-142.20,(\mathrm{C})$ & & \\
\hline $\begin{array}{c}8.94, \mathrm{~S}, 1 \mathrm{H}, \mathrm{CH} \\
\mathrm{C}-149.12\end{array}$ & $\mathrm{C}-142.20,(\mathrm{C})$ & $\mathrm{C}-150.24,(\mathrm{C})$ & & \\
\hline $\begin{array}{c}8.97, \mathrm{~S}, 1 \mathrm{H},(\mathrm{CH}) \\
\mathrm{C}-148.05\end{array}$ & $141.91,(\mathrm{C})$ & $\mathrm{C}-152.80,(\mathrm{C})$ & & \\
\hline
\end{tabular}




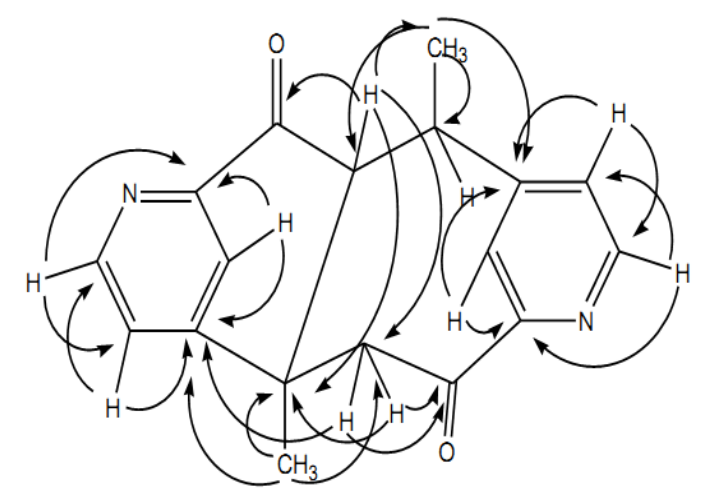

Figure 1. HMBC correlations $(\mathrm{H} \rightarrow \mathrm{C})$ for $\mathbf{1}$

The condensation of pyridine with a cyclooktane skeleton was determined by the ${ }^{1} \mathrm{H}$ -

Compound 1. The ${ }^{1} \mathrm{H}$ NMR, $(600 \mathrm{MHz}$, $\mathrm{CDCl}_{3}$ ): see Table 1. $\delta 8.94,(\mathrm{~s}, 1 \mathrm{H}, \mathrm{H}-15)$; 87.46, (d, 1H, J=4.8 Hz, H-12); $\delta 7.56,(\mathrm{~d}, 1 \mathrm{H}$, $\mathrm{J}=4.8 \mathrm{~Hz}, \mathrm{H}-5) ; \delta 3.41,(\mathrm{~m}, \mathrm{H}-14) ; \delta 2.66,(\mathrm{~d}, 1 \mathrm{H}$, $\mathrm{J}=3.6 \mathrm{~Hz}, \mathrm{H}-14 \mathrm{a}) ; \delta 8.97$, (s, 1H, H-16); $\delta 2.54$, (d, $1 \mathrm{H}, \mathrm{J}=18 \mathrm{~Hz}, \mathrm{H}-7 \mathrm{a}) ; \delta 2.81$, (d, $1 \mathrm{H}, \mathrm{J}=18 \mathrm{~Hz}$, $\mathrm{H}-7 \mathrm{~b}) ; \delta 8.70,(\mathrm{~d}, 1 \mathrm{H}, \mathrm{J}=4.8 \mathrm{~Hz}, \mathrm{H}-11) ; \delta 8.75$, $(\mathrm{d}, 1 \mathrm{H}, \mathrm{J}=4.8 \mathrm{~Hz}, \mathrm{H}-4)$ and a methyl resonances at $\delta 1.52,\left(\mathrm{~d}, 3 \mathrm{H}, \mathrm{J}=7.2 \mathrm{~Hz}, \mathrm{CH}_{3} 18\right)$ and $\delta 1.67$, (s, 3H, $\left.\mathrm{CH}_{3}, 17\right) .{ }^{13} \mathrm{C}$ NMR: $\delta 204.91$ (C-1); $\delta 141.91$ (C-2); $\delta 149.03$ (C-4); $\delta 116.18$ (C-5); $\delta 152.80$ (C-6); $\delta 44.21$ (C-6a); $\delta 48.97$ (C-7); $\delta 203.70$ (C-8); $\delta 142.20$ (C-9); $\delta 148.97$ (C-11); $\delta 116.10$ (C-12); $\delta 150.24$ (C-13); $\delta 36.19$ (C-14); $\delta 63.25$ (C-14a); $\delta 149.12(\mathrm{C}-15) ; \delta 148.05$ (C$16) ; \delta 26.97$ (C-17); $\delta 22.16$ (C-18). ESIMS $\mathrm{m} / \mathrm{z}$ $315[\mathrm{M}+\mathrm{Na}]^{+} ;$HRESIMS $m / z 315.1104[\mathrm{M}+$
$\mathrm{Na}]^{+}$(calcd for $\mathrm{C}_{18} \mathrm{H}_{16} \mathrm{~N}_{2} \mathrm{O}_{2} \mathrm{Na}, 315.32557$ ).

However, chemical shift of the proton 14a was observed at a weak magnetic field by $\Delta s=$ $0.11 \mathrm{ppm}$. The structure of compound 2 was determined as (14aR)-6a,14-dimethyl6a,7,14,14a-tetrahydro-2,6:9,13-di (metheno) azocino[5,4-e]azecine-1,8-dione. This is stereoisomer of the compound $\mathbf{1}$ and named "moncaryopterine B", (2).

Compound 2. The ${ }^{1} \mathrm{H}$ NMR (600 MHz, $\left.\mathrm{CDCl}_{3}\right)$ : see Table $1 . \delta 8.87,(\mathrm{~s}, 1 \mathrm{H}, \mathrm{H}-15) ; \delta 7.47,(\mathrm{~d}, 1 \mathrm{H}$, $\mathrm{J}=4.8 \mathrm{~Hz}, \mathrm{H}-4) ; \delta 2.99,(\mathrm{~m}, \mathrm{H}-14) ; \delta 2.77,(\mathrm{~d}, 1 \mathrm{H}$, $\mathrm{J}=3.6 \mathrm{~Hz}, \mathrm{H}-14 \mathrm{a}) ; \delta 9.02$, (s, $1 \mathrm{H}, \mathrm{H}-16) ; \delta 7.58$, $(\mathrm{d}, 1 \mathrm{H}, \mathrm{J}=4.8 \mathrm{~Hz}, \mathrm{H}-5) ; \delta 2.49,(\mathrm{~d}, 1 \mathrm{H}, \mathrm{J}=18 \mathrm{~Hz}$, H-7a); $22.60,(\mathrm{~d}, 1 \mathrm{H}, \mathrm{J}=18 \mathrm{~Hz}, \mathrm{H}-7 \mathrm{~b}) ; \delta 8.70$, (d, $1 \mathrm{H}, \mathrm{J}=4.8 \mathrm{~Hz}, \mathrm{H}-11)$; $\delta 8.76,(\mathrm{~d}, 1 \mathrm{H}, \mathrm{J}=4.8 \mathrm{~Hz}, \mathrm{H}-$ $12)$; and a methyl resonances at $\delta 1.85,(\mathrm{~s}, 3 \mathrm{H}$, $\left.\mathrm{CH}_{3}, 17\right)$ and $\delta 1.30,\left(\mathrm{~d}, 3 \mathrm{H}, \mathrm{J}=7.2 \mathrm{~Hz}, \mathrm{CH}_{3}, 18\right)$ and. ${ }^{13} \mathrm{C}$ NMR: $\delta 205.32$ (C-1); $\delta 141.85$ (C-2); $\delta 148.08$ (C-4); $\delta 116.38$ (C-5); $\delta 152.33$ (C-6); $\delta 43.96$ (C-6a); $\delta 48.24$ (C-7); $\delta 203.68$ (C-8); $\delta 142.11$ (C-9); $\delta 148.99$ (C-11); $\delta 116.19$ (C-12); $\delta 150.23$ (C-13); $\delta 36.16$ (C-14); $\delta 62.19$ (C-14a); $\delta 149.43(\mathrm{C}-15) ; \delta 149.00(\mathrm{C}-16) ; \delta 27.58(\mathrm{C}-$ 17); $\delta 21.87(\mathrm{C}-18)$. ESIMS $m / z 315[\mathrm{M}+\mathrm{Na}]^{+}$; HRESIMS $m / z$ 315.1111 $\left[\mathrm{M}+\mathrm{Na}^{+}\right.$(calcd for $\mathrm{C}_{18} \mathrm{H}_{16} \mathrm{~N}_{2} \mathrm{O}_{2} \mathrm{Na}$ ), 315.32557).

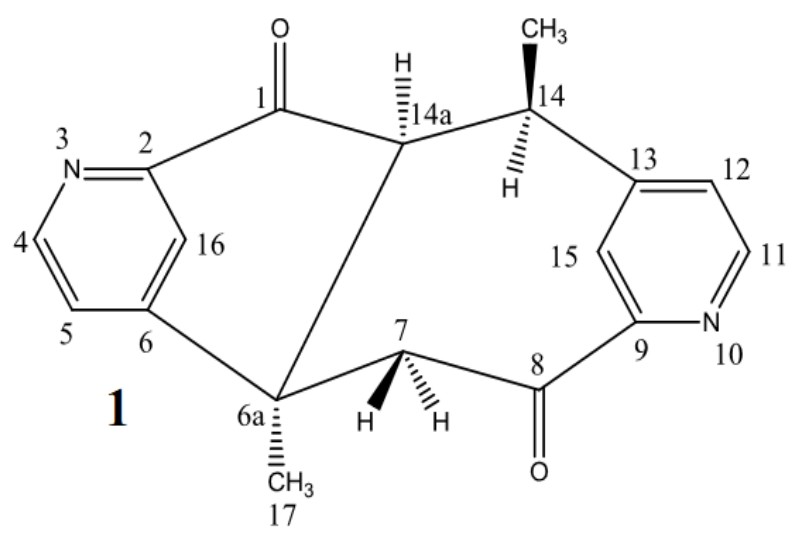

Moncaryopterine A

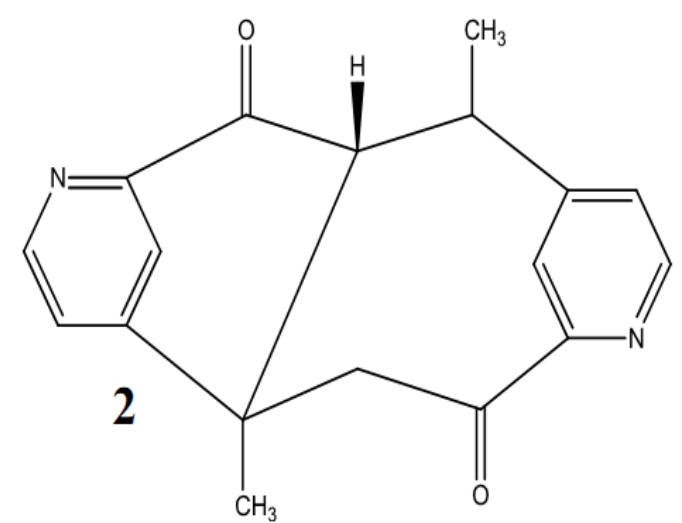

Moncaryopterine B 


\section{CONCLUSIONS}

We have isolated 2 alkaloids from the aerial parts of Caryopteris mongolica Bunge. grown in the Mongolian flora and molecular structures of them were elucidated by modern $1 \mathrm{D}$ and $2 \mathrm{D}$ NMR methods. These are naturally a new alkaloids isolated and identified for the first time, thus given a trivial name "moncaryopterine-A"

“moncaryopterine-B” (2), respectively.

\section{ACKNOWLEDGEMENT}

This work was supported by the TWAS-CAS Fellowships for Visiting Scholars in China.

\section{REFERENCES}

1. V. I. Grubov (2008), Key to the vascular plants of Mongolia, Ulaanbaatar, pp. 254.

2. China's outstanding Doctoral Dissertation (2010), Study on Reproductive Biology of Caryopteris mongolica Bunge.

3. U. Ligaa (2006), Medicinal plants of Mongolia Used in Mongolian Traditional Medicine, Ulaanbaatar, pp. 210-211.

4. Yong Hong Zhang, Qing Yi Wei, Zhong Li Liu, Li Yang and Dong Liang Cheng (2004), Two New Steroidal Glycosides from Caryopteris terniflora, Chinese Chemical Letters Vol. 15, No12, pp.1445-1447.

5. Shatar. S., Adams R. P. (1999), The essential oil of Caryopteris mongolica Bunge. From Mongolia, J. Essent. Oil Bear. Plants. Vol. 2, No 1, pp.25-28.

6. G.G. Zapesochnaya and T. T. Pangarova (1973), Hypolactin-7-glucosiode from Caryopteris mongolica, Khimiya Prirodnych Soedinenii, No4, pp. 554.
7. Zhang, Yong-Hong,; Yang, Li; Cheng, Dong-Liang (2000), Iridoid glucosides from Caryopteris mongolica, Pharmazie, 55(11), pp. 845-847.

8. Zhang Y., Cheng D. (2001), Studies on the chemical constituents of Caryopteris mongolica, Lanzhou Dasue Suebao, Ziran Kesueban, Vol. 37, No 5, pp. 69-71.

9. Natural Resources of the Russia (2011), The studies of chemical constituents and biological activity from plants of Caprifoliaceae-Lobeliaceae-Berbenaceae family, Sant-Peterburg-Moskow, pp.174.(in Russian).

10. Zhao, Da Peng; Matsunami, Katsuyoshi; Otsuka, Hideaki (2009), Iridoid glucoside, (3R)-oct-1-en-3-ol glucosides, and phenylethanoid from the aerial parts Caryopteris incana Journal of Natural Medicines 63(3), pp.241-247.

11. Zhang, Yong-Hong; Wang, Yan-Li; Wei, Qing-Yi; Cai, Yu-Jun; Wang, Qin; Liu, Zhong-Li (2005), Diterpenoids from the Chinese herb Caryopteris terniflora and their antibacterial and antitumor activity, Pharmazie, 60(7), pp. 551-553.

12. Gao, Jian Jun; Han, Gui Qiu; Yang Li (1996), Two new phenylpropanoid glucosides from Caryopteris incana (Thumb.) Miq. Chinese Chemical Letters 7(5), pp. 445-448.

13. Matsumota, Takeshi; Mayer, C; Eugster, Conrad H. (1969), Helvetica Chemica Acta, 52(3), pp. 808-812.

14. Sebastien Hannedouche, Eduoard Stanislas, Claude Moulis, Isabella Fouraste' (2000), Iridoids from Caryopteris $X$ clandonensis, Phytochemistry 54, pp.807-809. 